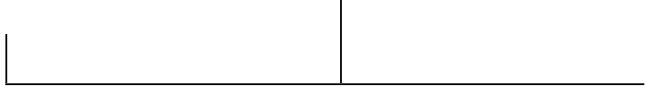

Rev. Latinoam. Psicopat. Fund., São Paulo, v. 11, n. 2, p. 278-285, junho 2008

\title{
Mortalidade por transtornos mentais e comportamentais e a reforma psiquiátrica no Brasil contemporâneo
}

Fernando Portela Câmara

A mortalidade por transtornos mentais e comportamentais têm aumentado no Brasil com a redução progressiva das internações hospitalares. A reforma psiquiátrica do governo aparentemente está contribuindo para este cenário preocupante, com sua política de desospitalização.

Palavras-chave: Mortalidade por transtornos mentais comportamentais, reforma psiquiátrica, política de saúde mental brasileira 
No Brasil moderno instituiu-se uma política governamental de desospitalização dos doentes mentais em substituição ao modelo para o qual não temos ainda nenhum indicador seguro de sua eficiência em resolver a demanda deste doentes e suas famílias, que buscam alívio e controle de seus padecimentos. $\mathrm{O}$ jornal O Globo, de dezembro de 2007, publicou em uma extensa reportagem duras críticas à política de saúde mental do governo inapropriadamente denominada "reforma psiquiátrica". Nesta matéria denunciava-se que o número de mortes de doentes mentais cresceu $41 \%$ nos últimos cinco anos, ao mesmo tempo em que $25 \%$ dos leitos psiquiátricos foram fechados sem a necessária implantação de serviços substitutivos.

Em face desta situação, a política da reforma psiquiátrica parece não ter progredido além do seu patamar ideológico. De fato, não notamos uma postura pragmática, de ação efetiva ou dados objetivos, que prove ser esta uma alternativa mais eficiente que a do atendimento hospitalar para os casos graves de transtornos mentais. Aparentemente, o modelo proposto pela reforma psiquiátrica se baseia no princípio de que o essencial do tratamento do doente mental é a reintegração social, proposta que tem sido criticada e matéria de controvérsia nos países em que foi implantada.

O melhor indicador para se avaliar esta política de desospitalização é a mortalidade na categoria dos transtornos mentais e comportamentais (capítulo V da CID-10) e sua correlação com 
a redução das internações. Para isso, usamos dados do DATASUS/Ministério da Saúde referentes ao período de 1996 a 2005 (não houve dados completos para os anos de 2006 e 2007 no momento em que este trabalho foi escrito).

A Figura 1 mostra as variações de mortalidade das categorias da CID-10 (v. tabela 1) entre os anos de 1996 e 2005, corrigidas para o aumento da população no mesmo período. Note-se que, ao mesmo tempo, as mortalidades de algumas categorias que integram parte das prioridades do SUS diminuíram. Entretanto, observa-se que a mortalidade por transtornos mentais e comportamentais foi a mais prevalente em todas as categorias da CID-10 no período estudado, com um alarmante aumento real de $62,3 \%$.

A Figura 2 mostra o gráfico do aumento da mortalidade no país na categoria dos transtornos mentais e comportamentais versus redução proporcional de internações (corrigida para o incremento da população brasileira), no período de 1996 a 2005 (retas determinadas por regressão linear). A correlação Pearson $r$ para ambas variáveis mostrou uma forte associação entre mortalidade e redução de leitos para internação $(\mathrm{r}=0,92, \mathrm{p}<0,001)$, e a relação entre mortalidade e redução proporcional de internações (corrigida) foi altamente significativa no modelo de regressão linear ( $\mathrm{p}<0,001$ para ambos coeficientes angular e linear), com um coeficiente de determinação ajustado $\mathrm{R}^{2}=83,1 \%$, mostrando que o modelo é preditivo e válido, com a redução proporcional de leitos explicando $83,1 \%$ da variância da variável resposta (mortalidade). O modelo obtido foi:

Mortalidade no país $=5201+9525$. (redução proporcional de internações)

Note que enquanto a variação da população brasileira sofreu um incremento médio de 1,17 vezes entre 1996 e 2005, a mortalidade sofreu um incremento médio de 1,90; portanto, um aumento real de $62,3 \%$.

Comparada à Rede de Saúde Suplementar (Figura 3), vemos também que a mortalidade proporcional na rede SUS foi significativamente maior (teste de MannWhitney, $\mathrm{p}=0,0327$ ). Os dados correspondem ao ano de 2004 , último registro completo do DATASUS no momento em que este trabalho foi escrito. A população de usuários da Saúde Suplementar era de 38.769.158, e a do Brasil 179.108.134, portanto, a população efetiva de usuários do SUS seria teoricamente a diferença, ou seja, 140.338.976. A mortalidade no SUS, por transtornos mentais e comportamentais, apresentou um risco de mortalidade 4,6 vezes maior que na Rede de Saúde Suplementar.

Em conclusão, houve um aumento real na mortalidade por transtornos mentais e comportamentais no período de 1996-2005, que corresponde aos dados completos disponíveis no DATASUS até a elaboração deste trabalho. Este aumento 
foi significativamente maior que a variação da população residente no período, correlacionou-se fortemente com a progressiva redução das internações psiquiátricas, e mostrou uma relação direta (modelo de regressão linear) com este processo. O aumento da mortalidade revelado pelo DATASUS é alarmante e aponta para a necessidade de uma revisão radical da atual política de saúde mental no Brasil.

\section{Referências}

O governo não quer saber de quem ouve vozes. $O$ Globo, Rio de Janeiro, 9 dezembro 2007, O país, p. 15.

\section{Base de dados consultada}

Agência Nacional de Saúde Suplementar, Informações Sobre Saúde Suplementar. Disponível em: <http://anstabnet.ans.gov.br/materia.htm>. Acesso em: 2 abr. 2004. Internações Hospitalares do SUS - Por Local de Internação - Brasil (Por Ano de Competência; Especialidade Psiquiatria). Ministério da Saúde - Sistema de Informações Hospitalares do SUS (SIH/SUS). Disponível em: <http://tabnet.datasus.gov.br/cgi/ tabcgi.exe?sih/cnv/rxuf.def $>$. Acesso em: 2 abr. 2004.

MS, DATASUS, População Residente-Brasil. Disponível em: <http:// tabnet.datasus.gov.br/cgi/deftohtm.exe?ibge/cnv/popuf.def>. Acesso em: 2 abr. 2004.

Óbito por Ocorrência por Ano de Óbito Segundo Capítulo CID-10. Fonte: MS/SVS/ DASIS - Sistema de Informações Sobre Mortalidade - SIM. Disponível em: $<$ http:// tabnet.datasus.gov.br/cgi/deftohtm.exe?sim/cnv/obtuf.def $>$. Acesso em: 2 abr. 2004.

Óbito por Ocorrência por Ano de Óbito Segundo Unidade da Federação (Capítulo V CID-10). Fonte: MS/SVS/DASIS - Sistema de Informações Sobre Mortalidade - SIM. Disponível em: <http://tabnet.datasus.gov.br/cgi/deftohtm.exe?sim/cnv/obtuf.def $>$. Acesso em: 2 abr. 2004. 
Tabela 1: Capítulos da CID-10 relacionados no DATASUS

\section{CAPÍTULOS DA CID-10}

I. Algumas doenças infecciosas e parasitárias

II. Neoplasias (tumores)

III. Doenças sangue órgãos hemat e transt imunitár

IV. Doenças endócrinas nutricionais e metabólicas

V. Transtornos mentais e comportamentais

VI. Doenças do sistema nervoso

VII. Doenças do olho e anexos

VIII. Doenças do ouvido e da apófise mastóide

IX. Doenças do aparelho circulatório

X. Doenças do aparelho respiratório

$\mathrm{Xl}$. Doenças do aparelho digestivo

XII. Doenças da pele e do tecido subcutâneo

XIII. Doenças sist osteomuscular e tec conjuntivo

XIV. Doenças do aparelho geniturinário

XV. Gravidez parto e puerpério

$\mathrm{XVl}$. Algumas afec originadas no período perinatal

XVII. Malf cong deformid e anomalias cromossômicas

XVIII. Sint sinais e achad anorm ex clín e laborat

XX. Causas externas de morbidade e mortalidade 


\section{OBSERVANDO}

A PSIQUIATRIA

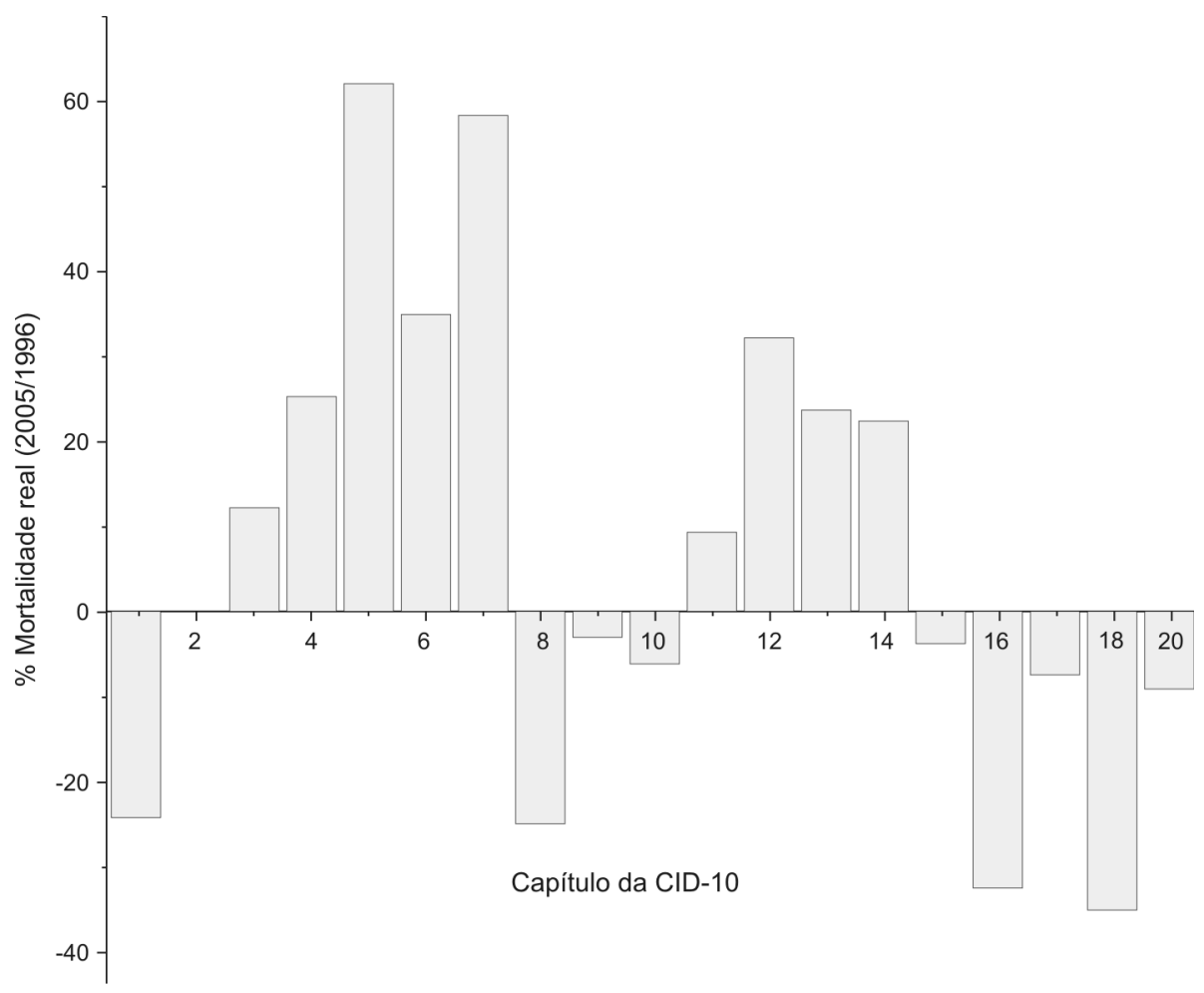

FIGURA 1

Variação da mortalidade por capítulo da CID-10 no período de 1996 a 2005.

Note que o aumento positivo da mortalidade por transtornos mentais e comportamentais foi o maior em todas as categorias. 


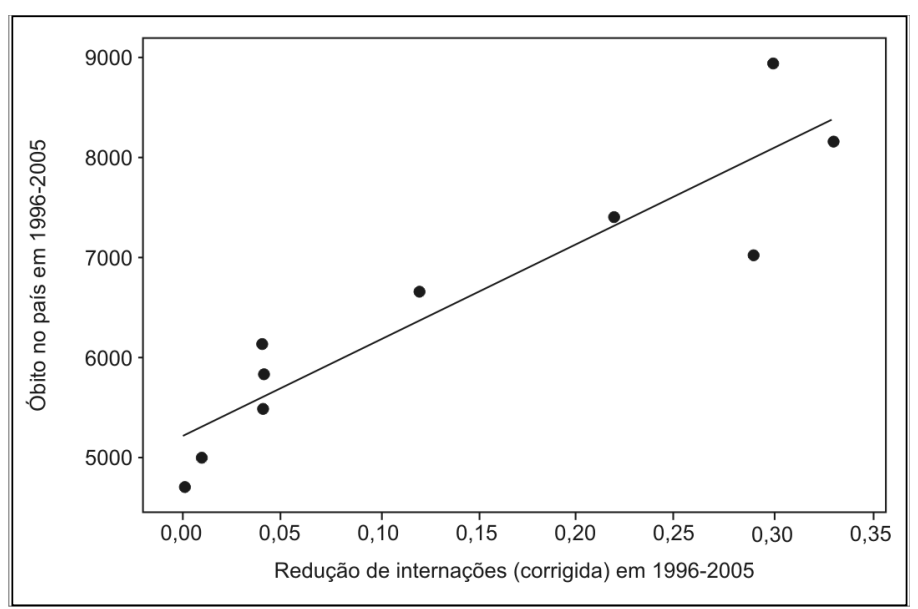

FIGURA 2

Aumento da mortalidade no país em função da redução das internações expressas em proporções corrigidas em função do incremento da população brasileira. A reta foi obtida por regressão linear $(p<0,001)$. A correlação Pearson $r$ foi $0,92(p<0,001)$

e a coeficiente de determinação $\left(R^{2}\right)$ foi $83,1 \%$.

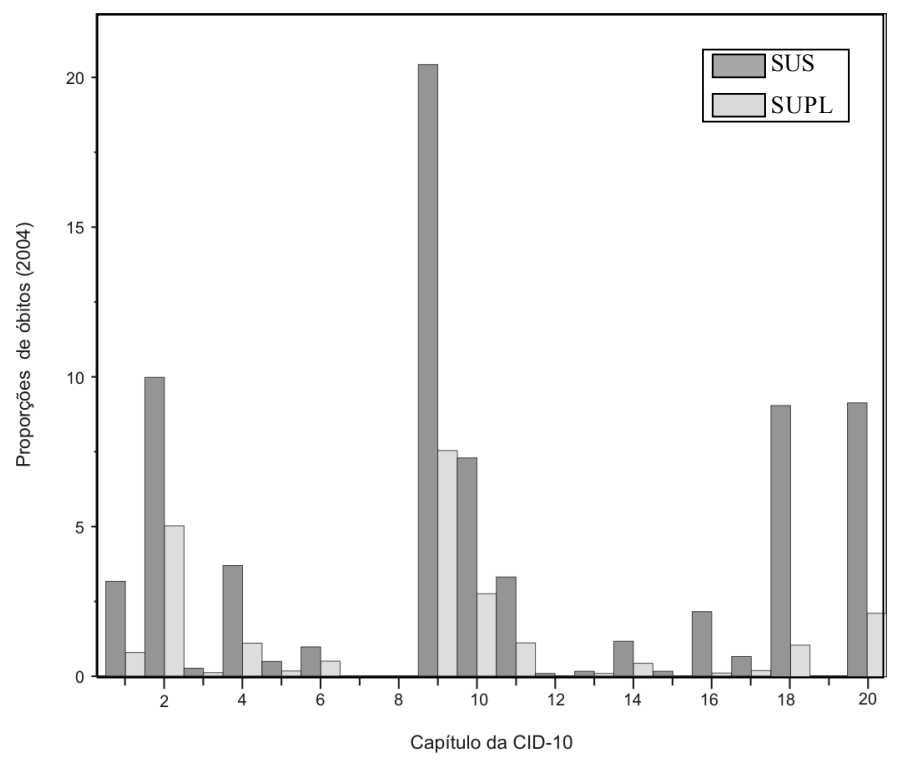

FIGURA 3

Mortalidade proporcional na rede SUS e de Saúde Suplementar por capítulo da CID-10. Note que os itens relativos a doenças de olhos e ouvidos não estão representados por serem insignificantes. 
(Mortalidad por los trastornos mentales y del comportamiento y la reforma psiquiátrica en el Brasil contemporáneo)

La mortalidad por trastornos mentales y del comportamiento ha aumentado en Brasil con la reducción gradual de las internaciones hospitalarias. La reforma psiquiátrica del gobierno aparentemente, está contribuyendo para ese escenario preocupante con su política de deshospitalización.

Palabras claves: Trastornos mental y del comportamiento, mortalidad por disturbio mental, reforma psiquiátrica, política de salud mental brasileña

(La mortalité par trouble mental et comportemental et la réforme psychiatrique au Brésil contemporain)

La mortalité par trouble mental et comportemental a augmenté au Brésil avec la réduction progressive des hospitalisations. La réforme psychiatrique du gouvernement contribue apparemment à ce cadre inquiétant, avec sa politique de deshospitalisation.

Mots clés: Trouble mental et comportemental, mortalité, réforme psychiatrique, politique brésilienne de santé mentale

(Death resulting from mental and behavioral disorders, and psychiatric reform in contemporary Brazil)

Death due to mental and behavioral disorders has increased in Brazil with the gradual reduction in the numbers of hospitalized mental patients. The federal government's psychiatric reform, based largely on closing down mental hospitals, is apparently contributing to this phenomenon.

Key words: Mental and behavioral disorders, death rates, psychiatric reform, mental health policies in Brazil

Versão inicial recebida em abril de 2008 Versão aprovada para publicação em abril de 2008

\section{Fernando Portela Câmara}

MD; PhD; Professor associado, SEDI-IMPPG-UFRJ - Setor de Epidemiologia de Doenças Infecciosas do Instituto de Microbiologia Professor Paulo de Góes, da Universidade Federal do Rio de Janeiro (Rio de Janeiro, RJ, Brasil).

Rua Pinheiro Machado, 25/405

22231-090 Rio de Janeiro, RJ, Brasil

Fone: (21) 9618-8603

e-mail: fp3camara@yahoo.com.br 